\title{
Competing Regression Models for Longitudinal Data
}

\author{
Airlane P. Alencar*,1, Julio M. Singer ${ }^{1}$, and Francisco M. M. Rocha ${ }^{2}$ \\ ${ }^{1}$ Universidade of São Paulo (Departamento de Estatística, Instituto de Matemática e Estatística, Caixa \\ Postal 66281, São Paulo, SP, 05314-970, Brazil) \\ ${ }^{2}$ Universidade Federal de São Paulo (Departamento de Ciência e Tecnologia - São José dos Campos, SP, \\ Brazil)
}

Received zzz, revised zzz, accepted zzz

\begin{abstract}
The choice of an appropriate family of linear models for the analysis of longitudinal data is often a matter of concern for practitioners. To attenuate such difficulties, we discuss some issues that emerge when analyzing this type of data via a practical example involving pretest-posttest longitudinal data. In particular, we consider log-normal linear mixed models (LNLMM), generalized linear mixed models (GLMM) and models based on generalized estimating equations (GEE). We show how some special features of the data, like a non-constant coefficient of variation, may be handled in the three approaches and evaluate their performance with respect to the magnitude of standard errors of interpretable and comparable parameters. We also show how different diagnostic tools may be employed to identify outliers and comment on available software. We conclude by noting that the results are similar, but that GEE-based models may be preferable when the goal is to compare the marginal expected responses.
\end{abstract}

Supplemental materials for this article are available on line.

Key words: Mixed models; Generalized linear models; Estimating equations method; Longitudinal data; Pretest/posttest measures;

\section{Introduction}

Mixed models are very useful to analyze data with a hierarchical structure, where measures from distinct units are independent and those from within units are correlated. In particular, they are appropriate to analyze repeated measures in longitudinal studies. The classical linear mixed model considered by Laird \& Ware (1982) is expressed as

$$
\mathbf{y}_{i}=\mathbf{X}_{i} \beta+\mathbf{Z}_{i} \mathbf{b}_{i}+\mathbf{e}_{i}, i=1, \ldots, N,
$$

where $\mathbf{y}_{i}$ is the $\left(n_{i} \times 1\right)$ vector of responses for the $i$-th unit, $\mathbf{X}_{i}$ is the $\left(n_{i} \times p\right)$ fixed effects specification matrix, $\beta$ is the corresponding $(p \times 1)$ vector of parameters, $\mathbf{b}_{i}$ is a $(q \times 1)$ vector of random effects with $\mathrm{E}\left(\mathbf{b}_{i}\right)=\mathbf{0}$ and $\operatorname{Var}\left(\mathbf{b}_{i}\right)=\mathbf{G}, \mathbf{Z}_{i}$ is a $\left(n_{i} \times q\right)$ specification matrix for the random effects, and $\mathbf{e}_{i}$ is a $\left(n_{i} \times 1\right)$ vector of random errors independent of $\mathbf{b}_{i}$, with $\mathrm{E}\left(\mathbf{e}_{i}\right)=\mathbf{0}, \operatorname{Var}\left(\mathbf{e}_{i}\right)=\mathbf{R}_{i}$, and $\mathbf{G}(q \times q)$ and $\mathbf{R}_{i}$ $\left(n_{i} \times n_{i}\right)$ are symmetric positive definite matrices. The marginal expected response is $\mathrm{E}\left(\mathbf{y}_{i}\right)=\mathbf{X}_{i} \beta$ and the conditional (on the individual effect $\left.\mathbf{b}_{i}\right)$ expected response is $\mathrm{E}\left(\mathbf{y}_{i} \mid \mathbf{b}_{i}\right)=\mathbf{X}_{i} \beta+\mathbf{Z}_{i} \mathbf{b}_{i}$. The inclusion of random effects may account for heterogeneity of regression coefficients across sample units, possibly due to several unmeasured factors that affect the response variable (Fitzmaurice et al. 2004).

It follows that

$$
\Sigma=\operatorname{Var}\left(\mathbf{y}_{i}\right)=\mathbf{Z}_{i} \mathbf{G} \mathbf{Z}_{i}^{\top}+\mathbf{R}_{i},
$$

Corresponding author: e-mail: lane@ime.usp.br, Phone: +55-11-30916218 
where the first and second summands correspond, respectively, to the inter- and intra-individual covariance structures. When $\mathbf{R}_{i}=\sigma^{2} \mathbf{I}$, the model is termed homoskedastc conditionally independent model and correlation among observations on the same unit $i$ arises from their sharing the unobservable (latent) variable, $\mathbf{b}_{i}$ (Diggle et al, 2002, p. 128).

In the original formulation, Laird \& Ware (1982) assumed that $\mathbf{b}_{i}$ and $\mathbf{e}_{i}$ in (1.1) follow normal distributions. Unbiased and consistent estimators of the variance components may be obtained by maximizing the restricted likelihood function and estimators of the fixed effects may be obtained via maximum likelihood (Jiang 2007). In practice, however, it is often not plausible to assume that the response is normally distributed. To bypass this problem, a possible strategy is to consider a log-normal linear mixed model (LNLMM), where the response is assumed to follow a multiplicative model with log-normal errors; the corresponding linearized model is additive and may be expressed as (1.1). For a broader choice of response distributions, two alternatives are generalized linear mixed models (GLMM) and models in which the parameters are estimated via generalized estimating equations (GEE).

In the GLMM approach, a distribution of the exponential family is considered for the response. In the usual formulation, individual observations are assumed to be independent conditionally on the random effects. In a more general setting, it is possible to consider different covariance structures. Estimation may be based on maximum likelihood (ML), penalized quasi-likelihood (PQL) or pseudo-likelihood methods (see Fitzmaurice et al. (2008) p.90-94 for details).

In the GEE-based approach, the form of the distribution of the vectors of responses $\left(\mathbf{y}_{i}\right)$ is not specified; the only assumption is that the marginal model depends only on the mean vector and on the covariance matrix of $\mathbf{y}_{i}$. Estimators are obtained as solutions to generalized estimating equations and are consistent even when the covariance structure is misspecified. In this case, the standard errors may not be correct, but valid standard errors can be obtained via the sandwich estimator (Liang \& Zeger 1986). Because GEEbased models do not belong to the class of mixed models, they do not allow the evaluation of individual effects.

Excellent surveys on linear and generalized linear mixed models are presented in Demidenko (2004) and Jiang (2007). Marginal models analyzed via GEE are carefully addressed in Hardin \& Hilbe (2002). Song (2007) and Fitzmaurice et al. (2008) present recent reviews on the all these models. Good references on longitudinal data analysis using mixed and marginal models are: Diggle et al. (2002), Fitzmaurice et al. (2004) and Molenberghs \& Verbeke (2005).

We compare some features of the three approaches by fitting LNLMM, GLMM and GEE-based models to data obtained from a pretest-posttest longitudinal study. We show how some important characteristics of the data may be incorporated in the three models, discuss the robustness of parameter estimators in the presence of outliers and comment on their computational implementation. In Section 2 we introduce the data along with a preliminary exploratory analysis with the objective of identifying models for the analysis. In Sections 3, 4 and 5, we present the results of fitting, respectively, LNLMM, GLMM and GEE-based models, detailing their specification, the estimation method and discussing diagnostic tools. In Section 6 we discuss some advantages, disadvantages, and properties of each family of models and present some concluding remarks.

\section{Preliminary analysis of the longitudinal pretest-posttest data}

We present a pretest-posttest longitudinal dataset, highlighting its distinctive characteristics and conduct a preliminary descriptive analysis. In particular, we evaluate coefficients of variation and fit a naive model using least squares in order to identify possible competing models.

A study conducted at the School of Dentistry of the University of São Paulo, Brazil, was designed to compare the efficiency in the removal of bacterial plaque under daily use of a low cost experimental (monoblock) toothbrush to that of a conventional toothbrush. In the study, 32 children, 4 to 6 years old, 
were randomly divided into 2 groups. Sixteen children received the monoblock toothbrush and the remaining children received the conventional toothbrush at the beginning of the study and used it for 45 days. During this period, data on a bacterial plaque index were collected before (pretest) and after (posttest) toothbrushing in sessions spaced by 15 days. The data is available in Nobre \& Singer (2007).

Scatter plots of the pretest and posttest bacterial plaque indices for each session and type of toothbrush are presented in Figure 1.

Figure 1: Scatter plots of pretest versus posttest bacterial plaque indices.

Insert Figure 1 approximately here.

The bacterial plaque indices measured before $(\mathrm{x})$ and after $(\mathrm{y})$ toothbrushing are supposed to have the following characteristics, suggested in Singer and Andrade (1997) which we include for the sake of selfcontainment:

a) A pretest plaque index equal to zero implies an expected posttest plaque index also equal to zero.

b) Pretest and posttest plaque indices are non-negative.

c) The data are possibly heteroskedastic (because the response is non-negative and follows the relation $\mathrm{E}(y) \leq x)$; i.e. the expected posttest bacterial plaque index (y) must be smaller than the pretest index $(\mathrm{x})$.

d) The relation between pretest and posttest plaque indices may be non-linear.

e) The observations carried out on the same child are possibly correlated.

For simplicity, we analyze the data conditionally on the values of the pretest observations. For an error-in-variables approach to a similar problem, the reader is referred to Aoki et al. (2003).

Following the suggestions of Singer \& Andrade (1997) and Singer et al. (2002), a model that satisfies (a)-(e) is a multiplicative model of the form

$$
y_{i j d}=\beta_{j d} x_{i j d}^{\gamma_{j d}} \varepsilon_{i j d},
$$

where $y_{i j d}$ is the posttest bacterial plaque index for the $i$-th individual using the $j$-th toothbrush in the $d$-th session, $x_{i j d}$ is the corresponding pretest index, $i=1, \ldots, 16, j=0$ (conventional), 1 (monoblock), $d=1,2,3,4, \beta_{j d}$ and $\gamma_{j d}$ are parameters to be estimated and $\varepsilon_{i j d}$ are non-negative random errors.

The efficiency of a toothbrush may be measured by the relative residual bacterial plaque index, defined as the expected ratio between the posttest and pretest bacterial plaque indices, say $\mathrm{E}\left(y_{i j d} / x_{i j d}\right)=$ $\beta_{j d} x_{i j d}^{\gamma_{j d}-1} \mathrm{E}\left(\varepsilon_{i j d}\right)$. With this definition, the smaller the relative residual bacterial plaque index, the more efficient is the toothbrush. Note that the measure of efficiency is proportional to $\beta_{j d}$ and it is equal to $\beta_{j d}$ when $\gamma_{j d}=1$ and $\mathrm{E}\left(\varepsilon_{i j d}\right)=1$. In this multiplicative model, if $\gamma_{j d}=1$, the efficiency does not depend on the pretest index, but when $\gamma_{j d}>1\left(\gamma_{j d}<1\right)$, the efficiency decreases (increases) according to the pretest index.

The characteristics (a)-(d) are clearly satisfied by model (2.1); in particular the heteroskedasticity for the response stems from $\operatorname{Var}\left(y_{i j d}\right)=\left(\beta_{j d} x_{i j d}^{\gamma_{j d}}\right)^{2} \operatorname{Var}\left(\varepsilon_{i j d}\right)$, even for homoskedastic errors $\left(\varepsilon_{i j d}\right)$. Requirement (e) may be incorporated in the specification of the error distribution. Model (2.1) may be linearized by taking logarithms, i.e.,

$$
\ln \left(y_{i j d}\right)=\lambda_{j d}+\gamma_{j d} \ln \left(x_{i j d}\right)+e_{i j d}
$$

where $\lambda_{j d}=\ln \left(\beta_{j d}\right)$ and $e_{i j d}=\ln \left(\varepsilon_{i j d}\right)$.

To identify whether random intercepts should be included, we started by fitting model (2.2) assuming uncorrelated errors. In Figure 2a, we plot the residuals versus the rank of the mean residual (mean of 
the four residuals for each child); some children have all four residuals larger than others, suggesting that there are subject-specific components affecting the response. This variability may be modelled by random intercepts. The individual profiles of $\ln (y)$ versus $\ln (x)$ plotted in Figure $2 \mathrm{~b}$ do not suggest that individual slopes are different so that random slopes may not be necessary. Standardized residuals are plotted versus fitted values in Figure 2c. Three outliers may be identified; they correspond to the second evaluation for the 12-th child in the conventional toothbrush group and for the third and fourth evaluation sessions of the 13-th child in the monoblock toothbrush group.

Figure 2: (a) Residuals versus ranks of subject mean residuals. (b) Individual profiles of $\ln (y)$ versus $\ln (x)$. (c) Residuals versus fitted values.

Insert Figure 2.

The analysis of the parameter estimates of model (2.2) also suggests that $\gamma_{j d}=1$. Under this restriction, the efficiency of a toothbrush in each session can be measured by the expected posttest/pretest bacterial plaque index ratio, $\mathrm{E}\left(y_{i j d} / x_{i j d}\right)=\beta_{j d} \mathrm{E}\left(\varepsilon_{i j d}\right)$. Since the corresponding standard deviation is $\mathrm{SD}\left(y_{i j d} / x_{i j d}\right)=\beta_{j d} \mathrm{SD}\left(\varepsilon_{i j d}\right)$, the coefficient of variation $\mathrm{CV}\left(y_{i j d} / x_{i j d}\right)=\mathrm{SD}\left(\varepsilon_{i j d}\right) / \mathrm{E}\left(\varepsilon_{i j d}\right)$ should be constant for all sessions and toothbrush groups if the errors $\varepsilon_{i j d}$ were identically distributed. Figure 3 suggests that the coefficients of variation of the ratios $y_{i j d} / x_{i j d}$ are different, and consequently that the standard deviations of the errors $\varepsilon_{i j d}$ in (2.1), may be larger for the conventional toothbrush in sessions 1 and 2 and for the monoblock toothbrush in sessions 3 and 4. Even after removing the 3 outliers suggested in Figure $2 \mathrm{c}$, the coefficients of variation still exhibit a similar behaviour.

Figure 3: Sample coefficients of variation of ratios between the posttest and pretest indices for each toothbrush and session for the complete and reduced (excluding 3 outliers) data sets.

Insert Figure 3.

The preliminary analysis suggests that the candidate models should include random intercepts, heteroskedasticity, and a larger coefficient of variation for the ratio posttest/pretest bacterial plaque indices for the conventional toothbrush in sessions 1 and 2 and for the monoblock toothbrush in sessions 3 and 4 .

\section{Analysis via log-normal linear mixed models}

Here we consider a LNLMM model of the form (2.2) that includes random effects to account for possible positive within-subjects correlations and also considers an error covariance matrix that incorporates the non-constant coefficients of variation identified in Section 2. Such models may be fitted via well established linear mixed model methodology, where the location and the covariance structure may be modeled separately.

The log-normal linear mixed model may be expressed as

$$
\mathbf{y}_{i j}^{*}=\mathbf{X}_{i j} \beta+\mathbf{1}_{4} b_{i j}+\mathbf{e}_{i j}, i=1, \ldots, 16, j=0,1,
$$

where $\mathbf{y}_{i j}^{*}=\left(\ln \left(y_{i j 1}\right), \ln \left(y_{i j 2}\right), \ln \left(y_{i j 3}\right), \ln \left(y_{i j 4}\right)\right)^{T}$, the specification matrices for the conventional and monoblock toothbrush groups $(\mathrm{j}=0,1)$ are respectively,

$$
\mathbf{X}_{i 0}=\left(\mathbf{I}_{4}, \mathbf{0}_{4}, \bigoplus_{d=1}^{4} \ln \left(x_{i j d}\right), \mathbf{0}_{4}\right), \mathbf{X}_{i 1}=\left(\mathbf{0}_{4}, \mathbf{I}_{4}, \mathbf{0}_{4}, \bigoplus_{d=1}^{4} \ln \left(x_{i j d}\right)\right)
$$

where $\bigoplus_{j=1}^{4} a_{i}$ denotes a diagonal matrix with the elements $a_{i}$ along the main diagonal, $\beta=\left[\lambda^{T} \gamma^{T}\right]^{T}$ is a $(16 \times 1)$ vector with $\lambda^{T}=\left[\lambda_{01}, \lambda_{02}, \lambda_{03}, \lambda_{04}, \lambda_{11}, \lambda_{12}, \lambda_{13}, \lambda_{14}\right], \gamma^{T}=\left[\gamma_{01}, \gamma_{02}, \gamma_{03}, \gamma_{04}, \gamma_{11}, \gamma_{12}, \gamma_{13}, \gamma_{14}\right]$, $\mathbf{1}_{4}=(1,1,1,1)^{T}$, and $\mathbf{0}_{4}$ denotes a $4 \times 4$ matrix with all elements equal to zero. The error vectors $\mathbf{e}_{i j}$ are 
independent and follow $N\left(\mathbf{0}, \mathbf{R}_{j}\right)$ distributions, where, as suggested by the preliminary analysis,

$$
\mathbf{R}_{j}=\bigoplus_{d=1}^{4} r_{j d}^{2}=\left\{\begin{array}{l}
\operatorname{diag}\left\{\tau_{1}^{2}, \tau_{1}^{2}, \tau_{2}^{2}, \tau_{2}^{2}\right\}, \text { if } j=0 \\
\operatorname{diag}\left\{\tau_{2}^{2}, \tau_{2}^{2}, \tau_{1}^{2}, \tau_{1}^{2}\right\}, \text { if } j=1
\end{array}\right.
$$

Also, we assume that the random effects, $b_{i j}$, are independent and follow $N\left(0, \sigma_{b}^{2}\right)$ distributions.

We present estimates of $\beta_{j d}^{*}=\mathrm{E}\left(y_{i j d} \mid x_{i j d}=1\right)$ instead of the estimates of $\beta_{j d}=\exp \left(\lambda_{j d}\right)$ in (2.1) as suggested in Singer et al. (2002). To compute these estimates, note that $\exp \left(b_{i}+e_{i j d}\right)$ has a log-normal distribution, so that $\mathrm{E}\left[\exp \left(b_{i j}\right)+e_{i j d}\right]=\exp \left[\left(\sigma_{b}^{2}+r_{j d}^{2}\right) / 2\right]$. This approach is more convenient for interpretation and comparison with other models. Expressions for estimators $\widehat{\beta}_{j d}^{*}$ and their corresponding asymptotic variances (obtained by the delta method) are presented in Table 1. Model (3.1)-(3.2) was fitted to the data using restricted maximum likelihood methods, available in SAS PROC MIXED (Littell et al. 2006). The codes and the data to fit the proposed models may be found on line in the Supplemental Materials link. The estimates are presented in Table 2.

Table 1: Expressions for the estimators of $\beta_{j d}^{*}=\mathrm{E}\left(y_{i j d} \mid x_{i j d}=1\right)$ and corresponding asymptotic variances. Insert Table 1.

Table 2: Estimates (Est.) and standard errors (SE) for saturated LNLMM, GLMM and GEE fitted to the complete and reduced data sets.

Insert Table 2.

Results obtained under this model were compared to those based on two other models of the form (3.1): the first with $\mathbf{R}_{j}=\operatorname{diag}\left(\tau_{j 1}^{2}, \ldots, \tau_{j 4}^{2}\right), j=0,1$, and the second with $\mathbf{R}_{j}=\tau^{2} \mathbf{I}_{4}$. This latter was the model adopted in Nobre \& Singer (2007) in their analysis and implies a constant coefficient of variation. The $\mathrm{AIC}$ and $\mathrm{BIC}$ obtained via restricted maximum likelihood for the three models were respectively (AIC= -96.8 and $\mathrm{BIC}=-92.4),(\mathrm{AIC}=-91.8, \mathrm{BIC}=-78.6)$ and $(\mathrm{AIC}=-74.9$ and $\mathrm{BIC}=-72.0)$, suggesting that $(3.1)-$ (3.2) is acceptable. The reader is referred to Jiang \& Rao (2003) for a detailed analysis of generalized information criteria and to Guerin \& Stroup (2000) or Gurka (2006) for a discussion on AIC and BIC for model comparison.

In order to evaluate the robustness of the estimators, the model was refitted to the reduced data obtained by ommiting the 3 outliers suggested in Figure 2c. The results are also presented in Table 2. The major discrepancies between the estimates for the complete and reduced data occur for the parameters $\beta_{j d}^{*}$ and $\gamma_{j d}$ for $(j, d)=(0,2),(1,3),(1,4)$ which correspond to the treatments with identified outliers. As expected, the estimated error variance $\left(\widehat{\sigma_{b}^{2}}\right)$ is smaller for the reduced data set.

Following the suggestions of Nobre \& Singer (2007), studentized conditional residual plots are presented in Figure 4 for both the complete and reduced data; the two outlying observations in Figure 4a correspond to large decreases in the posttest index relatively to the pretest index in the second session for the 12-th child in the conventional toothbrush group and in the fourth session for the 13-th child in the monoblock toothbrush group. It is worth noting that fitting the model with $\mathbf{R}_{j}=\tau^{2} \mathbf{I}_{4}$, as in Nobre \& Singer (2007), these 2 outliers are more apparent in the residual analysis; furthermore an extra outlier is detected in the third session for a child in the monoblock group (as in Figure 2c). The model that includes (3.2) accommodates this observation and seems to be more suitable. The QQ plots in Figures 5a (complete data) and $5 \mathrm{~b}$ (ommiting possible outliers) suggest mild deviations from the normality assumption, but we do not believe that this could jeopardize the results.

Figure 4: Studentized conditional residuals versus subject indices for LNMM. (a) Complete data. (b) Reduced data.

Insert Figure 4.

Figure 5: QQ plot of studentized conditional residuals for LNMM. (a) Complete data. (b) Reduced data. 
Insert Figure 5.

We did not identify evidence against the hypothesis that $\gamma_{j d}=1, j=0,1, d=1,2,3,4$, under model (3.1)-(3.2) $(\mathrm{p}=0.310)$. The estimates of the parameters for this model are displayed in Table 3. Note that Nobre \& Singer (2007) did not verify whether $\gamma_{j d}=1$.

Table 3: Estimates (Est.) and standard errors (SE) for saturated LNLMM, GLMM and GEE fitted to the complete and reduced data sets assuming $\gamma_{j d}=1$.

Insert Table 3.

The conventional toothbrush seems more efficient than the monoblock toothbrush as suggested in Figure 6. P-values for interaction and main effects tests are presented in Table 4. The difference in the expected efficiencies of the two types of toothbrush is not the same for all sessions $(\mathrm{p}=0.010)$ : for the monoblock toothbrush it is constant along the sessions $(\mathrm{p}=0.387)$ but this is not so for the conventional toothbrush $(\mathrm{p}=0.006)$. The expected efficiencies are different for the monoblock and conventional toothbrushes in sessions 1 and 2 ( $\mathrm{p}=0.021$ and $\mathrm{p}<0.001$, respectively) and are similar in sessions 3 and 4 ( $\mathrm{p}=0.266$ and $\mathrm{p}=0.840$ ). This suggests that the monoblock toothbrush is less efficient only in sessions 1 and 2 and is as efficient as the conventional toothbrush in sessions 3 and 4. In Nobre \& Singer (2007), under the constant coefficient of variation assumption, the interaction effects were not significant.

Figure 6: Estimated relative residual bacterial plaque index and corresponding 95\% confidence intervals based on the LNLMM (complete data set).

Insert Figure 6.

Table 4: P-values for interaction and main effects tests for LNLMM, GLMM and GEE. Insert Table 4.

\section{Analysis via generalized linear mixed models}

In this section, we consider a GLMM based on a gamma distribution. As the LNLMM, this model is appropriate for a non-negative variable like the posttest bacterial plaque index and meets all the requirements set forth in Section 2. A positive correlation among the repeated measures is induced by the inclusion of random effects.

The typical gamma generalized linear mixed model with a logarithmic link function for the posttest bacterial plaque indices, $\mathbf{y}_{i j}=\left(y_{i j 1}, \ldots, y_{i j 4}\right)^{\top}$, may be specified as

$$
\begin{aligned}
& y_{i j d} \mid b_{i j} \sim \operatorname{Gamma}\left(\mu_{i j d}, \phi\right), \\
& \mu_{i j}=\mathrm{E}\left(\mathbf{y}_{i j} \mid b_{i j}\right), \quad \ln \left(\mu_{i j}\right)=\mathbf{X}_{i j} \beta+\mathbf{1}_{4} b_{i j}, \quad b_{i j} \sim N\left(0, \sigma_{b}^{2}\right),
\end{aligned}
$$

with $\mu_{i j}=\left(\mu_{i j 1}, \ldots, \mu_{i j 4}\right)^{\top}$ and $\operatorname{Var}\left(\mathbf{y}_{i j}\right)=\phi \mathbf{A}_{\mu_{i j}}$, where $\phi$ is a dispersion parameter, $\mathbf{A}_{\mu_{i j}}=\operatorname{diag}\left(\mu_{i j 1}^{2}, \mu_{i j 2}^{2}, \mu_{i j 3}^{2}, \mu_{i j 4}^{2}\right)$ and $\mathbf{X}_{i j}$ and $\beta$ defined in (3.1). In this context, the coefficient of variation of the ratio of the posttest bacterial plaque index to the pretest bacterial plaque index is constant for all treatments. Since this disagrees with our previous conclusions, we consider an alternative GLMM, maintaining (4.2) and replacing (4.1) with

$$
\operatorname{Var}\left(\mathbf{y}_{i j} \mid b_{i j}\right)=\mathbf{A}_{\mu_{i j}}^{1 / 2} \mathbf{W}_{i j} \mathbf{A}_{\mu_{i j}}^{1 / 2},
$$

where, by taking $\mathbf{W}_{i j}=\mathbf{R}_{j}$ as in (3.2), we include the heteroskedastic pattern identified in Figure 3. If we let $\mathbf{W}_{i j}=\phi \mathbf{I}$, it follows that (4.3) is less restrictive than (4.1) since it does not require $y_{i j d} \mid b_{i j}$ to follow gamma distribution as pointed by Jiang (2007). 
We estimated the parameters in (4.2)-(4.3) using the restricted pseudo-likelihood method presented in Wolfinger \& O'Connell (1993) and implemented in the PROC GLIMMIX of SAS (Littell et al. 2006). This method is based on a first-order Taylor approximation to $\ln \left(\mathbf{y}_{i j}\right)$ around $\mu_{i j}$. In order to maximize the restricted pseudo-likelihood function, a Newton-Raphson ridge optimization technique is considered.

Estimates of $\beta_{j d}^{*}=\mathrm{E}\left(y_{i j d} \mid x_{i j d}=1\right)=\mathrm{E}\left[\mathrm{E}\left(y_{i j d} \mid b_{i}, x_{i j d}=1\right)\right]$ as well as of the other parameters in the GLMM are presented in Table 2. Also, there is no evidence against the hypothesis $\gamma_{j d}=1(\mathrm{p}=0.441)$ and estimates of the parameters of model (4.2)-(4.3) with $\gamma_{j d}=1$ are presented in Table 3.

The studentized conditional residuals in Figure 7 present the same pattern as the corresponding residuals obtained for the LNLMM displayed in Figure 4.

Figure 7: Studentized conditional residuals for the GLMM model versus subject indices. (a) Complete data. (b) Reduced data.

Insert Figure 7.

\section{Analysis via generalized estimating equations based models}

Here we analyze the pretest-posttest data via models where the marginal distribution is not completely specified; such models depend only on a dispersion parameter and on a vector of parameters $(\theta)$ that indexes the mean $\mu_{i}(\theta)$ and the variance $\mathbf{V}_{i}(\theta)$. Estimates of the parameters are obtained as solutions to the generalized estimating equations

$$
\mathbf{S}(\beta)=\sum_{i=1}^{K} \frac{\partial \mu_{i}^{\top}}{\partial \beta} \Sigma_{i}^{-1}\left(\mathbf{y}_{i}-\mu_{i}(\beta)\right)=\mathbf{0}
$$

where $\Sigma_{i}=\phi \operatorname{diag}\left(\mathbf{V}_{i}\right)^{1 / 2} \mathbf{W}(\alpha) \operatorname{diag}\left(\mathbf{V}_{i}\right)^{1 / 2}$ and $\mathbf{W}$ is a working covariance matrix that incorporates the within subject correlation. If we set $V_{i}\left(\mu_{i}\right)=\mu_{i}^{2}$ and $\mathbf{W}=\mathbf{I}$, the solution is equivalent to a GLM with a gamma distribution and a log link function.

For the sake of comparison, we consider a GEE-based model for which

$$
\mu_{i j}=\mathrm{E}\left(\mathbf{y}_{i j}\right), \ln \left(\mu_{i j}\right)=\mathbf{X}_{i j} \beta, \operatorname{Corr}\left(y_{i j d}, y_{i j d^{\prime}}\right)=\alpha, d \neq d^{\prime}
$$

where $\mathbf{X}$ and $\beta$ are defined in (3.1) and $\phi_{j d}$ are dispersion parameters. To capture the behaviour of the coefficients of variation identified in Figure 3, the dispersion parameter may depend on the type of toothbrush $(j)$ and session $(d)$, i.e.,

$$
\phi_{j d}=\left\{\begin{array}{l}
\phi, \text { if }(j=0 \text { and } d=3 \text { or } 4) \text { or } \quad(j=1 \text { and } d=1 \text { or } 2) \\
\phi+\phi_{1}, \text { otherwise. }
\end{array}\right.
$$

This model was fitted via the $R$ package geepack (Halekoh et al. 2006) using GEE that allows for different covariates and even different link functions and estimating equations for the mean, scale and correlations as considered in Yan \& Fine (2004). The estimates of $\beta_{j d}^{*}$ and of the other parameters of model (5.2)-(5.3) are presented in Table 2. Again, no evidence against the hypothesis $\gamma_{j d}=1$ ( $\left.\mathrm{p}=0.481\right)$ was detected and the estimates under this restriction are presented in Table 3.

Plots of the studentized residuals are discussed in Venezuela et al. $(2007,2011)$ as well as Vens and Ziegler(2011). These residuals are presented in Figure 8 and their pattern are similar to those in Figure 4. These authors also show how to compute Cook's distance and a leverage measure in this setting. These quantities are also presented in Figure 8. The outlying observations are the same identified previously. Although, these observations do not present high leverage (Figure 8b), the corresponding Cook's distances are large for these outliers. Moreover, excluding them, no other observation is identified as an outlier.

Figure 8: Residual analysis for the GEE-based model with complete data. (a) Studentized conditional residuals. (b) Cook's distance. (c) Leverage measure for each observation. (d) Leverage measure for each subject. 
Insert Figure 8.

\section{Discussion}

We considered three families of linear models that are sufficiently flexible to accommodate different characteristics of longitudinal data. In particular, they may account for non-constant coefficients of variation, a feature that may justify differences generated by underlying log-normal and gamma distributions, as discussed in Wiens (1999).

Differences between estimates obtained under marginal and conditional models may occur due to noncomparable parameters, as indicate Lee \& Nelder (2004) and Fitzmaurice et al. (2008). In our example, the main goal was to evaluate the efficiency of two types of toothbrushes along 45 days. This may not be carried out by a direct comparison of the estimates of $\beta_{j d}$ in models (3.1)-(3.2), (4.2)-(4.3) and (5.2)(5.3), because such parameters have different interpretations. Assuming the correct specification of all models, the estimates of the original parameters $\beta_{j d}$ were smaller for LNLMM than for GLMM or GEEbased models. To bypass this problem, we compared the population averaged effects that correspond to the relative residual bacterial plaque indices, $\beta_{j d}^{*}=\mathrm{E}\left(y_{j d} \mid x_{j d}=1\right)$. For both LNLMM and GLMM, computation of this marginal expectation requires integration of the random effects in $\mathrm{E}\left[\mathbf{Y}_{i} \mid \mathbf{b}_{i}\right]$ while GEEbased models allow a direct estimation, an advantage reported by Serroyen et al. (2009), specially for non-linear mixed models.

In general, the estimates of the fixed parameters and their corresponding standard errors obtained under LNLMM, GLMM or GEE-based models are very close to each other, both with or without the exclusion of the outliers. The estimates of the variance components are also similar for LNLMM and GLMM. The estimate of $\tau_{1}^{2}$ is the most affected by the outliers because it is related to the extra-variance for the treatments where the outliers occurred. For the marginal model (GEE-based), the estimated correlation of repeated measures is smaller for the data without outliers and so is its standard error. The conclusions of all tests are the same for the three approaches, although the p-values are in general smaller for GEE-based models (see Table 4).

For random-effects models, like LNLMM and GLMM, another important issue is the robustness of estimation of fixed effects with respect to misspecification of the distribution of the random effects as discussed in Fitzmaurice et al. (2008). Verbeke \& Lesaffre (1997) showed that maximum likelihood estimators of fixed effects obtained for a linear mixed model under Gaussian distributed random effects are consistent even when the random effects are non-Gaussian. Also, restricted maximum likelihood estimators of the variance components are consistent; this is important because they are used to obtain estimates of both $\beta_{j d}^{*}$ and of its variance. Moreover, Verbeke \& Lesaffre (1996) also comment that it is not possible to assess the distribution of the random effects by analyzing the predicted $\widehat{b}_{i}$, what turns estimation robustness even more important. For GLMM, Litière et al. (2008) conclude that misspecification of the distribution of random effects may produce biased maximum likelihood estimators of fixed effects but that this bias is small when the variability of random effects is small; however, as the estimators of this variability are severely biased, it is not possible to evaluate whether the biases of fixed effect estimators are negligible or not. An additional advantage of GEE-based models is that GEE provide consistent estimators of fixed effects if the corresponding model is correctly specified regardless of the correlation and scale structures (Liang $\&$ Zeger 1986). Also, when the scale parameter depends on covariates, the corresponding estimators are consistent if the fixed effects and scale structures are correctly specified (Yan and Fine, 2004). Therefore, estimates of the fixed effects are more robust under GEE-based models and LNLMM than under GLMM if the covariance structure is misspecified.

Diagnostic tools are well developed for LNMM (see Nobre \& Singer (2007), for example). For GLMM, Vonesh et al. (1996) propose a goodness-of-fit statistic to evaluate the adequacy of an assumed mean and covariance structure and an approximate pseudo-likelihood test for the adequacy of the covariance structure; Xiang et al. (2002) use Cook's distance for clustered data to identify influential clusters and Zhu \& 
Lee (2003) propose a different method to measure influence for GLMM. Also, Waagepetersen (2006) assesses the goodness-of-fit of the random effects distribution using simulation. Nevertheless, more research is needed for residual analysis for GLMM as already indicated in Dean \& Nielsen (2007). Diagnostic tools for the GEE are presented, for example, in Venezuela et al. (2007), Venezuela et al. (2011) and Vens $\&$ Ziegler (2011) to detect influential and outlying observations using the projection (hat) matrix, Cook's distance, standardized residuals and half-normal plots of absolute residuals with simulated envelopes, the latter only for constant scale parameter as proposed in Park \& Shin (1998). Some diagnostic tools and references are presented in Table 5.

Table 5: Diagnostic Tools and References. Insert Table 5.

Other estimation issues involving the GEE method are related to missing data problems and the estimation of the correlation matrix. For example, Lu et al. (2009) show that fixed-effect estimators of LMM are biased under departures from normality in the presence of missing data. Also, based on simulation, they conclude that other (weighted and augmented weighted) robust estimators based on GEE provide valid inference for skewed non-Gaussian data when missing data follows a missing at random pattern. Sun et al. (2009) compare estimation methods for the correlation in the GEE framework and conclude that the degree of imbalance and variability in the temporal spacing of measurements, the value of the correlation and the type of outcome affect the choice of the best method.

A practical issue to be addressed is the availability of computational software to fit the families of models under consideration. Although we used R only to fit GEE-based models, there are libraries to fit GLMM (MASS with the command glmmPQL using penalized quasi-likelihood) and LNMM (lme4 and nlme). Table 6 presents procedures of SAS and R libraries to estimate the studied models.

Table 6: Available statistical packages using SAS and R and estimation methods used in this study. Insert Table 6.

If on the one hand estimates obtained under LNMM are robust to misspecification of random effects distributions, on the other, GEE-based models may be preferable for computational reasons, when the main goal is only to estimate and compare marginal expected responses. LNMM may be preferable to estimate subject-specific expected responses since a variety of diagnostic analyses are available for this family. To help users with respect to the choice of appropriate families of models, estimation procedures, software and diagnostic tools, we summarize different characteristics for the three approaches in Table 5. Other estimation methods are available for example in Fitzmaurice et al. (2008).

Finally, we mention that because of the relatively small (but not uncommon in studies of this type) sample size (32 children with 4 observations/child), the conclusions based on asymptotic results should be viewed with caution. A good practice in such cases is to fit different models to the data, use different diagnostic tools to detect possible outliers and verify whether the results are coherent. In our case, the three models generated similar results even when the possible outliers were included, suggesting that the conclusion seem pertinent. 

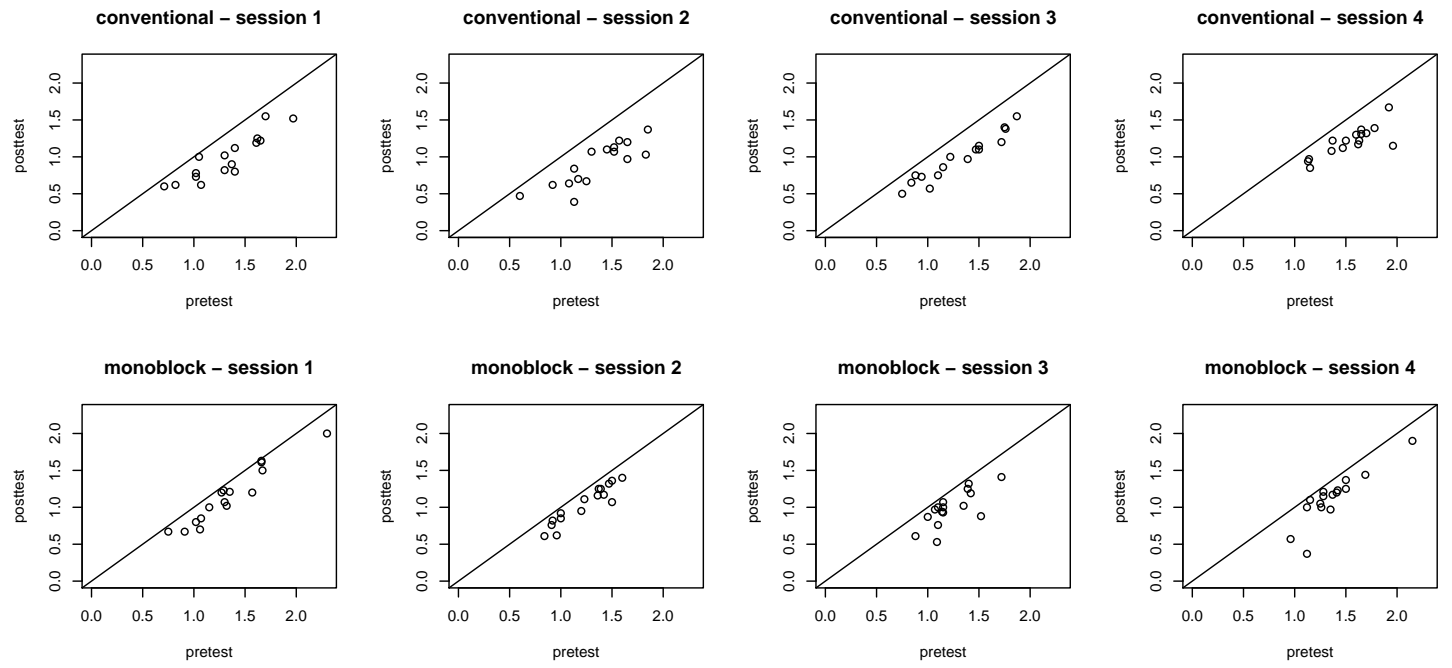

Figure 1 Scatter plots of pretest versus posttest bacterial plaque indices.

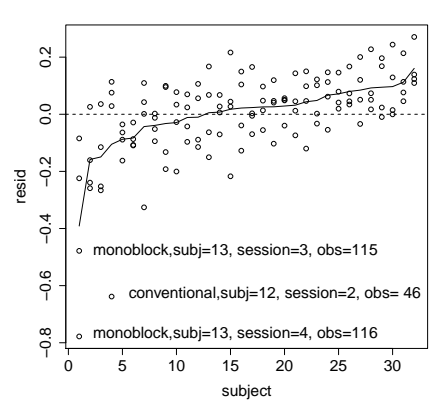

(a)

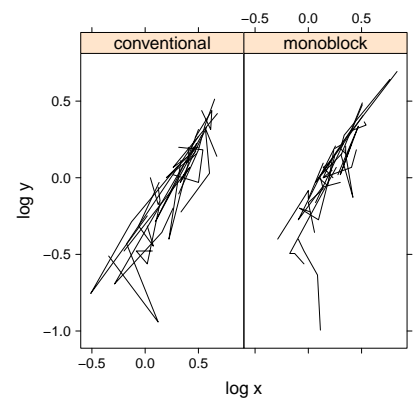

(b)

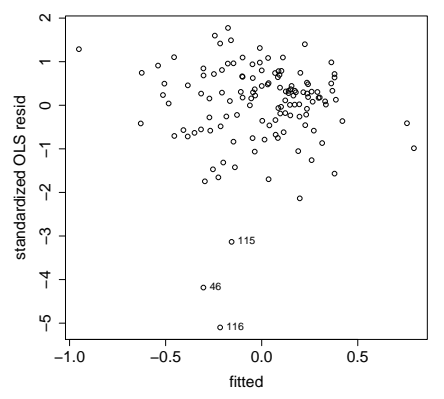

(c)

Figure 2 (a) Residuals versus ranks of subject mean residuals. (b) Individual profiles of $\ln (y)$ versus $\ln (x)$. (c) Residuals versus fitted values. 


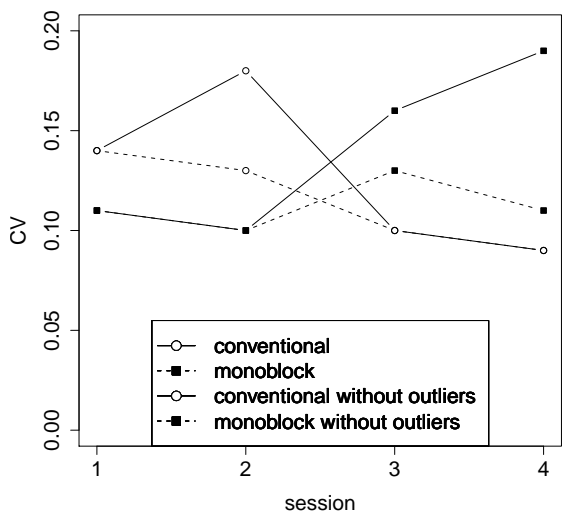

Figure 3 Sample coefficients of variation of ratios between the posttest and pretest indices for each toothbrush and session for the complete and reduced (excluding 3 outliers) data sets.

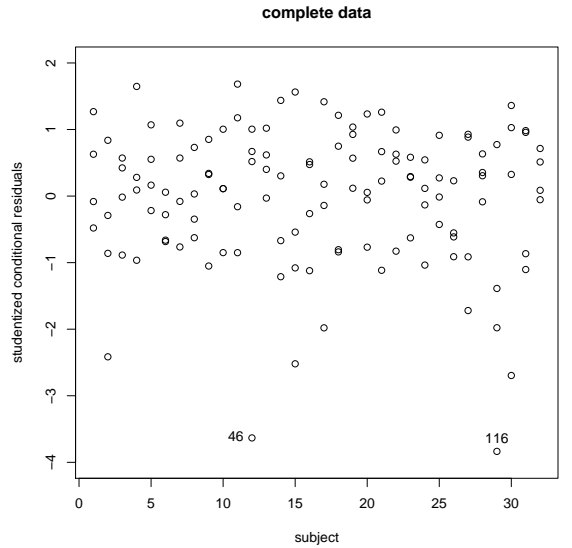

(a)

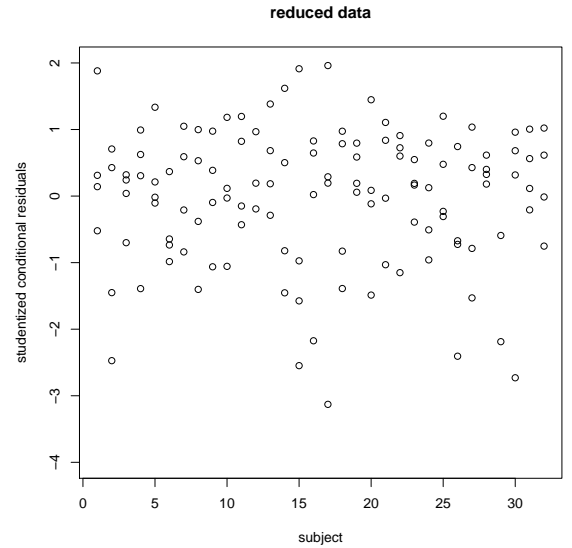

(b)

Figure 4 Studentized conditional residuals versus subject indices for LNMM. (a) Complete data. (b) Reduced data. 


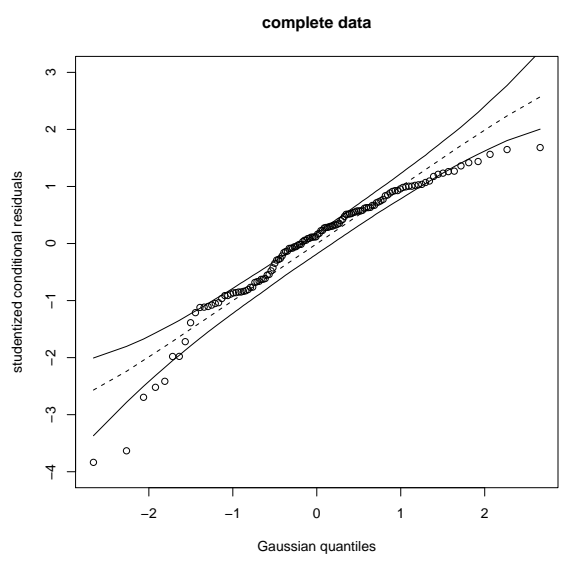

(a)

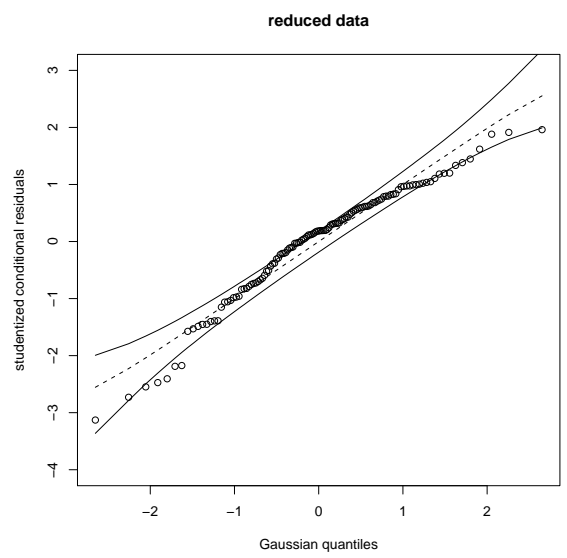

(b)

Figure 5 QQ plot of studentized conditional residuals for LNMM. (a) Complete data. (b) Reduced data.

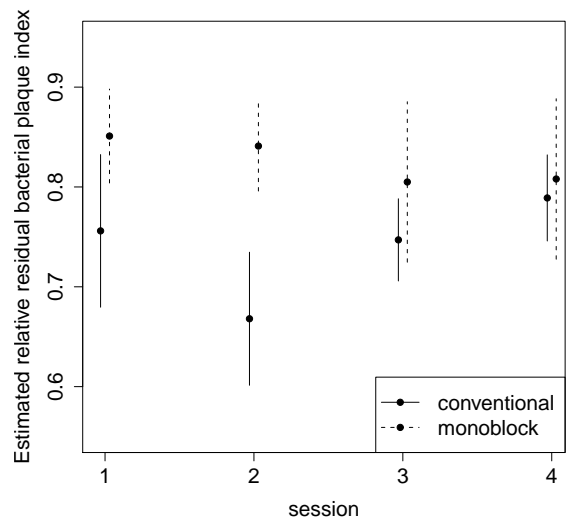

Figure 6 Estimated relative residual bacterial plaque index and corresponding 95\% confidence intervals based on the LNLMM (complete data set). 


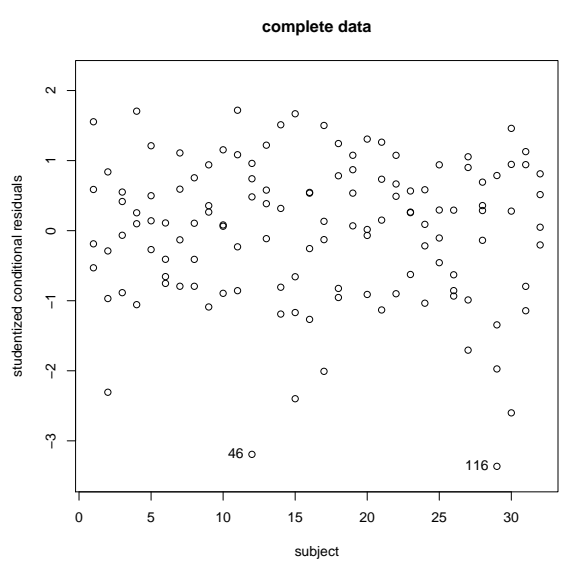

(a)

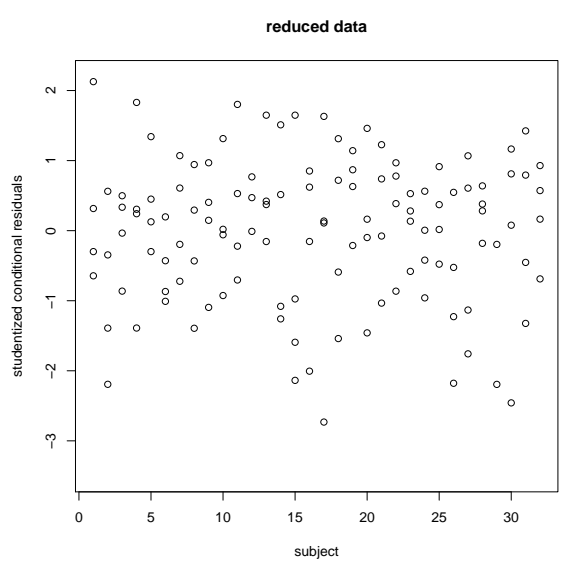

(b)

Figure 7 Studentized conditional residuals for the GLMM model versus subject indices. (a) Complete data. (b) Reduced data. 


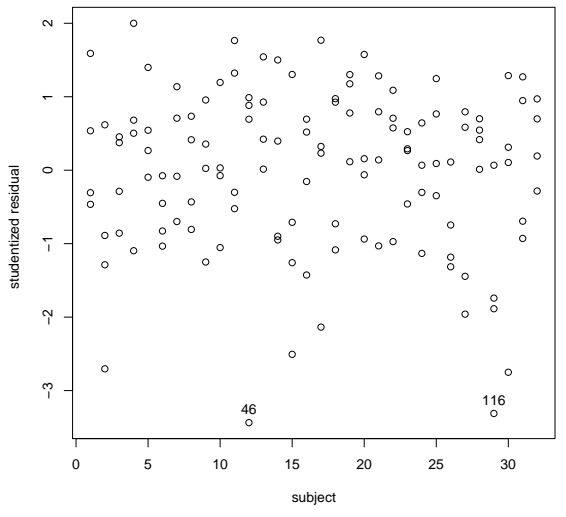

(a)

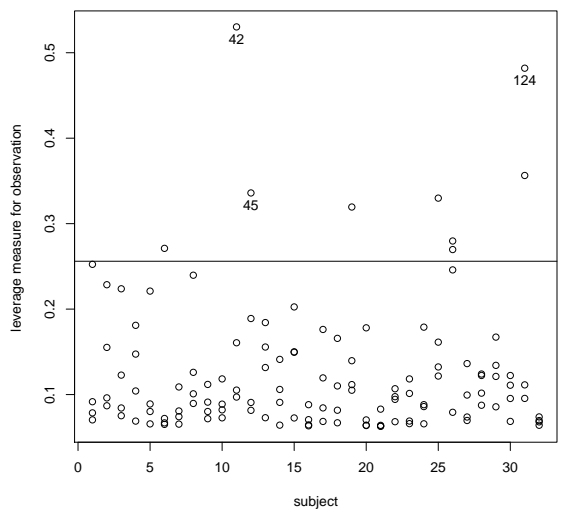

(c)

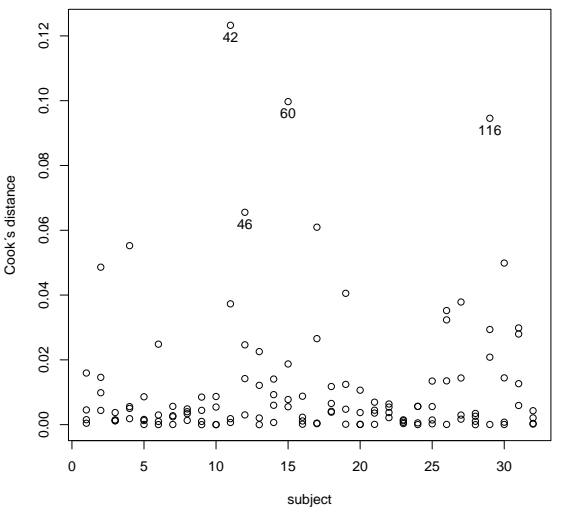

(b)

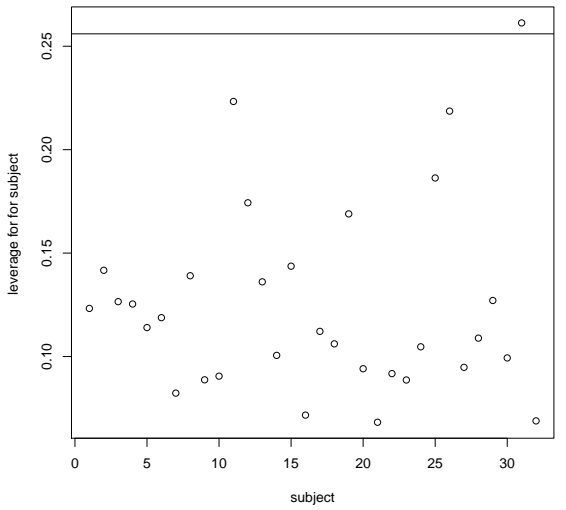

(d)

Figure 8 Residual analysis for the GEE-based model with complete data. (a) Studentized conditional residuals. (b) Cook's distance. (c) Leverage measure for each observation. (d) Leverage measure for each subject. 
Table 1. Expressions for the estimators of $\beta_{j d}^{*}=\mathrm{E}\left(y_{i j d} \mid x_{i j d}=1\right)$ and corresponding asymptotic variances

\begin{tabular}{ccc}
\hline Model & $\widehat{\beta}_{j d}^{*}$ & $\operatorname{Var}\left(\widehat{\beta}_{j d}^{*}\right)$ \\
\hline LNLMM & $\widehat{\beta}_{j d} \exp \left[0.5\left(\widehat{\sigma_{b}^{2}}+\widehat{r}_{j d}^{2}\right)\right]$ & $\left(\beta_{j d}^{*}\right)^{2}\left[\operatorname{Var}\left(\widehat{\lambda}_{j d}\right)+0.25\left(\sigma_{b}^{2}+r_{j d}^{2}\right)\right]$ \\
GLMM & $\widehat{\beta}_{j d} \exp \left(0.5 \widehat{\sigma}_{b}^{2}\right)$ & $\left(\beta_{j d}^{*}\right)^{2}\left[\operatorname{Var}\left(\widehat{\lambda}_{j d}\right)+0.25\left(\sigma_{b}^{2}\right)\right]$ \\
GEE & $\widehat{\beta}_{j d}$ & $\left(\beta_{j d}^{*}\right)^{2}\left[\operatorname{Var}\left(\widehat{\lambda}_{j d}\right)\right]$ \\
\hline
\end{tabular}




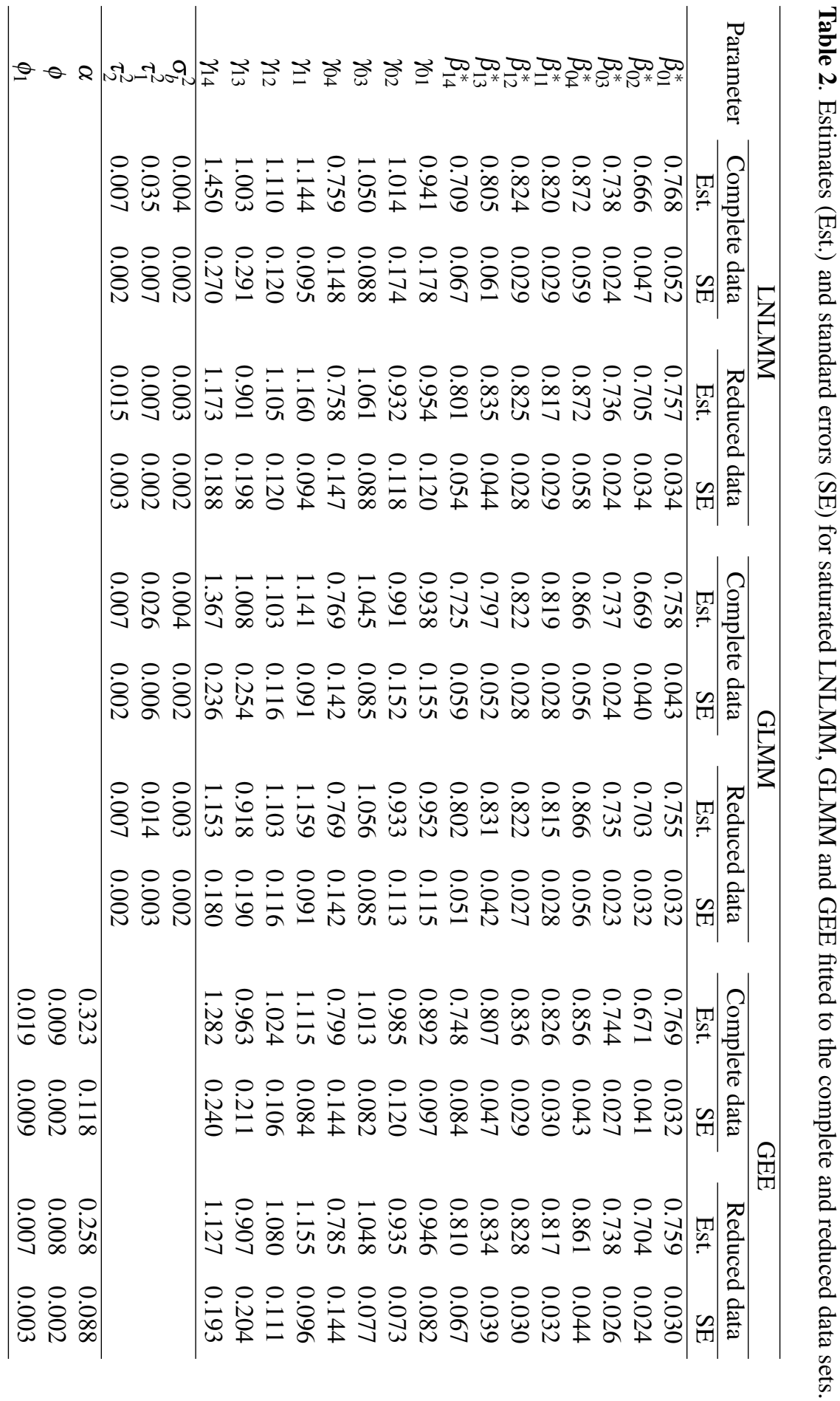




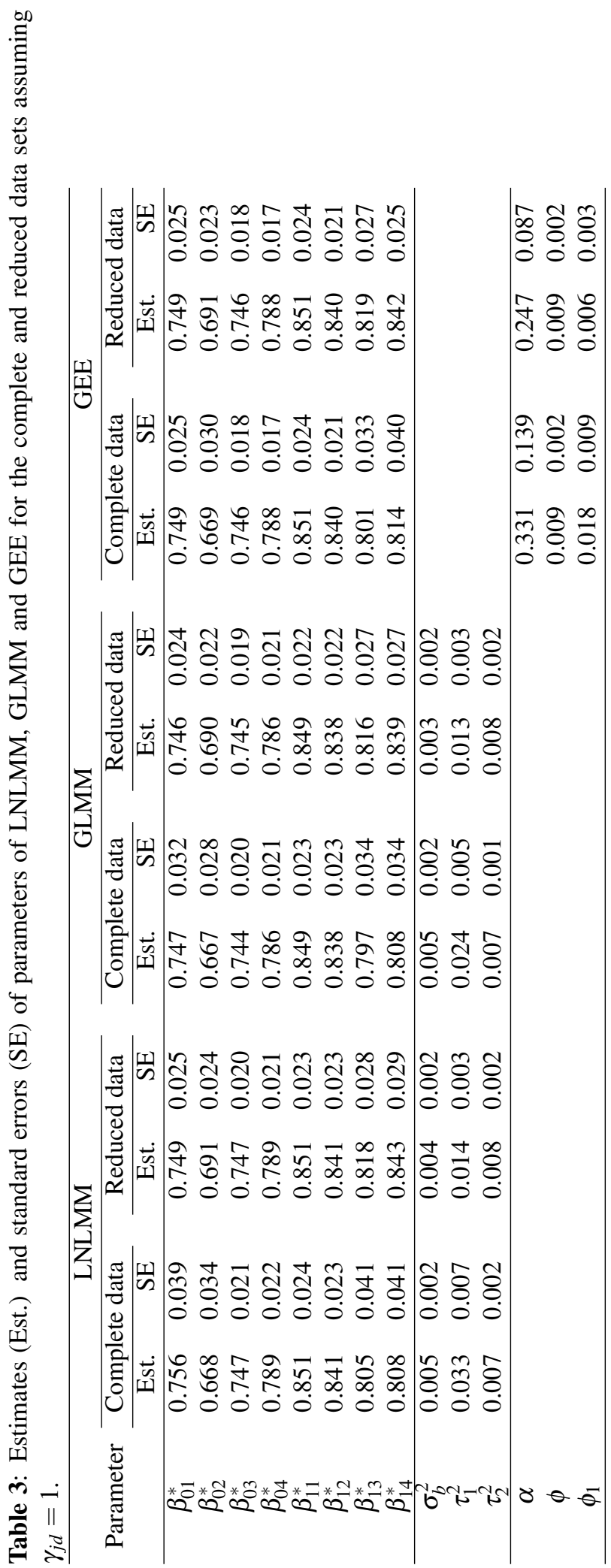


Table 4: P-values for interaction and main effects tests for LNLMM, GLMM and GEE.

\begin{tabular}{|c|c|c|c|c|c|c|c|}
\hline \multirow[b]{2}{*}{ Model } & \multirow{2}{*}{$\begin{array}{c}\text { Interaction } \\
\text { Effect }\end{array}$} & \multicolumn{2}{|c|}{ Session Effect } & \multicolumn{4}{|c|}{ Toothbrush Effect } \\
\hline & & Conventional & Monoblock & Session 1 & Session 2 & Session 3 & Session 4 \\
\hline LNLMM & 0.010 & 0.006 & 0.387 & 0.021 & $<0.001$ & 0.266 & 0.840 \\
\hline GLMM & 0.011 & 0.004 & 0.432 & 0.013 & $<0.001$ & 0.173 & 0.597 \\
\hline GEE & 0.009 & 0.004 & 0.544 & 0.004 & $<0.001$ & 0.127 & 0.545 \\
\hline
\end{tabular}

Table 5. Diagnostic Tools and References.

\begin{tabular}{ccc}
\hline Model & Diagnostic Tools & \multicolumn{1}{c}{ Reference } \\
\hline LNLMM & Conditional and marginal residuals and EBLUP & Nobre \& Singer (2007) \\
& Generalized leverage matrix & Nobre \& Singer (2011) \\
& Cook's distance & Tan et al. (2001) \\
\hline GLMM & Goodness-of-fit tests & Vonesh et al. (1996) \\
& Cook's distance & Xiang et al. (2002) \\
& Influence & Zhu \& Lee (2003) \\
& Goodness-of-fit for random effect & Waagepetersen (2006) \\
\hline GEE & Standardized residuals, projection matrices, Cook's distance & Venezuela et al. (2007) \\
& Local influence & Venezuela et al. (2011) \\
& Standardized residuals, projection matrices, Cook's distance & Vens \& Ziegler (2011) \\
\hline
\end{tabular}

Table 6. Available statistical packages using SAS and R and estimation methods used in this study.

\begin{tabular}{cccc}
\hline Model & SAS PROC & R libraries & Estimation method \\
\hline LNLMM & MIXED & lme4 and nlme & REML (Littell et al. 2006) \\
GLMM & GLIMMIX & MASS (glmmPQL) & Pseudo likelihood (Wolfinger \& O'Connell 1993) \\
GEE & GENMOD & geepack & GEE (Halekoh et al. 2006) \\
\hline
\end{tabular}

\section{Acknowledgements}

This research was supported by grants from the Conselho Nacional de Desenvolvimento Científico e Tecnológico (CNPq) and Fundação de Amparo à Pesquisa do Estado de São Paulo (FAPESP), Brazil. The authors also acknowledge the constructive comments and suggestions of the anonymous referee and the associate editor.

\section{Conflict of Interest}

The authors have declared no conflict of interest.

\section{Supplemental Materials}

Data file: Pretest and posttest bacterial plaque indices, type of toothbrush and session. (.txt file, .csv file)

SAS and R Programs: Programs to fit the models LNLMM (Proc Mixed/SAS), GLMM (Proc Glim$\mathrm{mix} / \mathrm{SAS}$ ) and GEE (library geepack/R) and the codes for all Figures (.txt file) 


\section{References}

Aoki, R., Achcar, J. A., Bolfarine, H. \& Singer, J. M. (2003), 'Bayesian analysis of null intercept errorsin-variables regression for pretest/posttest data', Journal of Applied Statistics 30, 3-12.

Dean, C. B. \& Nielsen, J. D. (2007), 'Generalized linear mixed models: a review and some extensions', Lifetime data analysis 13, 497-512.

Demidenko, E. (2004), Mixed models theory and applications, Wiley, New York.

Diggle, P., Liang, K. \& Zeger, S. (2002), Analysis of longitudinal data, Oxford University Press, Oxford.

Fitzmaurice, G. M., Davidian, M., Verbeke, G. \& Molenberghs, G. (2008), Longitudinal Data Analysis: A Handbook of Modern Statistical Methods, Chapman \& Hall/CRC, Boca Raton.

Fitzmaurice, G. M., Laird, N. \& Ware, J. (2004), Applied Longitudinal Analysis, Wiley, New York.

Guerin, L. \& Stroup, W. W. (2000), A simulation study to evaluate proc mixed analysis of repeated measures data, in 'Proceedings of the 12th Annual Conference on Applied Statistics in Agriculture', Kansas State University, Manhattan, pp. 170-203.

Gurka, M. J. (2006), 'Selecting the best linear mixed model under reml', The American Statistician 60, 1926.

Halekoh, U., Hojsgaard, S. \& Yan, J. (2006), 'The r package geepack for generalized estimating equations', Journal of Statistical Software 15, 1-11.

Hardin, J. W. \& Hilbe, J. M. (2002), Generalized estimating equations, Chapman and Hall/CRC, Boca Raton.

Jiang, J. (2007), Linear and generalized linear mixed models and their applications, Springer, New York.

Jiang, J. \& Rao, J. S. (2003), 'Consistent procedures for mixed linear model selection', Sankhyā 65, 23-42.

Laird, N. \& Ware, J. (1982), 'Random-effects models for longitudinal data', Biometrics 38, 963-974.

Lee, Y. \& Nelder, J. A. (2004), 'Conditional and marginal models: another view', Statistical Science 19, 219-238.

Liang, K. Y. \& Zeger, S. L. (1986), 'Longitudinal data analysis using generalized linear models', Biometrika 73, 13-22.

Litière, S., Alonso, A. \& Molenberghs, G. (2008), 'The impact of a misspecified random-effects distribution on the estimation and the performance of inferential procedures in generalized linear mixed models', Statistics in Medicine 27, 3125-3144.

Littell, R. C., Milliken, G. A., Stroup, W. W., Wolfinger, R. D. \& Schabenberger, O. (2006), SAS for mixed models, SAS Institute Inc, Cary.

Lu, N., Tang, W., He, H., Yu, Q., Crits-Christoph, P Zhang, H. \& Tu, X. (2009), 'On the impact of parametric assumptions and robust alternatives for longitudinal data analysis', Biometrical Journal 51, 627-643.

Molenberghs, G. \& Verbeke, G. (2005), Models for discrete longitudinal data, Springer, New York.

Nobre, J. S. \& Singer, J. M. (2007), 'Residual analysis for linear mixed models', Biometrical Journal 49, 1-13.

Nobre, J. S. \& Singer, J. M. (2011), 'Leverage analysis for linear mixed models', Journal of Applied Statistics 38, 1063-1072.

Park, C. G. \& Shin, D. S. (1998), 'An algorithm for generating correlated random variables in a class os infinitely divisible distributions', Journal of Statistical Computation and Simulation 61, 127-139.

Serroyen, J., Molenberghs, G., Verbeke, G. \& Davidian, M. (2009), 'Nonlinear models for longitudinal data', The American Statistician 63, 378-388.

Singer, J. M. \& Andrade, D. F. (1997), 'Regression models for the analysis of pretest/posttest data', Biometrics 53, 729-735. 
Singer, J. M., Nobre, J. S. \& Sef, H. C. (2002), 'Regression models for pretest/posttest data in blocks', Statistical Modelling 4, 1-15.

Song, P. X. K. (2007), Correlated data analysis - modelling, analystics and applications, Springer, New York.

Sun, W., Shults, J. \& Leonard, M. (2009), 'A note on the use of unbiased estimating equations to estimate correlation in analysis of longitudinal trials', Biometrical Journal 51, 5-18.

Tan, F. E. S., Ouwens, M. J. N. \& Berger, M. P. F. (2001), 'Detection of influential observations in longitudinal mixed effects regression models', Journal of the Royal Statistical Society: Series D (The Statistician) 50, 271-284.

Venezuela, M. K., Botter, D. A. \& Sandoval, M. C. (2007), 'Diagnostic techniques in generalized estimating equations', Journal of Statistical Computation and Simulation 77, 879-888.

Venezuela, M. K., Sandoval, M. C. \& Botter, D. A. (2011), 'Local influence in generalized estimating equations', Computational Statistics and Data Analysis 55, 1867-1883.

Vens, M. \& Ziegler, A. (2011), 'Generalized estimating equations and regression diagnostics for longitudinal controlled clinical trials: a case study', Computational Statistics and Data Analysis $\mathbf{x}, \mathbf{x}-\mathbf{x}$.

Verbeke, G. \& Lesaffre, E. (1996), 'A linear mixed-effects model with heterogeneity in the random-effects population', Journal of the American Statistical Association 91, 217-221.

Verbeke, G. \& Lesaffre, E. (1997), 'The effect of misspecifying the random-effects distribution in linear mixed models for longitudinal data', Computational Statistics and Data Analysis 53, 541-556.

Vonesh, E. F., Chinchilli, V. M. \& Pu, K. (1996), 'Goodness-of-fit in generalized nonlinear mixed-effects models', Biometrics 52, 572-587.

Waagepetersen, R. (2006), 'A simulation-based goodness-of-fit test for random effects in generalized linear mixed models', Scandinavian Journal of Statistics 33, 721-731.

Wiens, B. L. (1999), 'When log-normal and gamma models give different results: a case study', The American Statistician 53, 89-93.

Wolfinger, R. \& O’Connell, M. (1993), 'Generalized linear mixed models: A pseudo-likelihood approach', Journal of Statistical Computing and Simulation 48, 233-243.

Xiang, L., Tse, S. K. \& Lee, A. (2002), 'Influence diagnostics for generalized linear mixed models: applications to clustered data', Computational Statistics and Data Analysis 40, 759-774.

Yan, J. \& Fine, J. (2004), 'Estimating equations for association structures', Statistics in Medicine 23, 859874.

Zhu, H. T. \& Lee, S. Y. (2003), 'Local influence for generalized linear mixed models', The Canadian Journal of Statistics 31, 293-309. 